\title{
Darboux transformations and global explicit solutions for nonlocal Davey-Stewartson I equation
}

\author{
Zi-Xiang Zhou \\ School of Mathematical Sciences, Fudan University, \\ Shanghai 200433, China \\ Email: zxzhou@fudan.edu.cn
}

\begin{abstract}
For the nonlocal Davey-Stewartson I equation, the Darboux transformation is considered and explicit expressions of the solutions are obtained. Like the nonlocal equations in $1+1$ dimensions, many solutions may have singularities. However, by suitable choice of parameters in the solutions of the Lax pair, it is proved that the solutions obtained from seed solutions which are zero and an exponential function of $t$ respectively, by a Darboux transformation of degree $n$ are global solutions of the nonlocal Davey-Stewartson I equation. The derived solutions are soliton solutions when the seed solution is zero, in the sense that they are bounded and have $n$ peaks, and "line dark soliton" solutions when the seed solution is an exponential function of $t$, in the sense that they are bounded and their norms change fast along some straight lines.
\end{abstract}

\section{Introduction}

In [1], Ablowitz and Musslimani introduced the nonlocal nonlinear Schrödinger equation and got its explicit solutions by inverse scattering. Quite a lot of work were done after that for this equation and other equations. [2, 3, 4, 5, 6, 7, 8, 9, 10, 11]

In [12], Fokas studied high dimensional equations and introduced the nonlocal DaveyStewartson I equation

$$
\begin{aligned}
& \mathrm{i} u_{t}=u_{x x}+u_{y y}+2 \sigma u^{2} \bar{u}^{*}+2 u w_{y}, \\
& w_{x x}-w_{y y}=2 \sigma\left(u \bar{u}^{*}\right)_{y}
\end{aligned}
$$

where $w$ satisfies $\bar{w}^{*}=w$. Here $\bar{f}(x, y, t)=f(-x, y, t)$ for a function $f, *$ refers to complex conjugation. The solution of (1) is PT symmetric in the sense that if $(u(x, y, t), w(x, y, t))$ 
is a solution of (11), then so is $\left(u^{*}(-x, y,-t), w^{*}(-x, y,-t)\right)$. This leads to a conserved density $u \bar{u}^{*}$, which is invariant under $x \rightarrow-x$ together with complex conjugation.

As is known, the usual Davey-Stewartson I equation does not possess a Darboux transformation in differential form. Instead, it has a binary Darboux transformation in integral form. 13, 14 However, for the nonlocal Davey-Stewartson I equation (11), we can construct a Darboux transformation in differential form. Like the nonlocal equations in 1+1 dimensions, the solutions may have singularities. Starting from the seed solutions which are zero and an exponential function of $t$, we prove that the derived solutions can be globally defined and bounded for all $(x, y, t) \in \mathbf{R}^{3}$ if the parameters are suitably chosen. Unlike the usual Davey-Stewartson I equation where localized solutions are dromion solutions if the seed solution is zero, [15, 16, 17] the derived solutions here are soliton solutions in the sense that there are $n$ peaks in the solutions obtained from a Darboux transformation of degree $n$. If the seed solution is an exponential function of $t$, the norms of the derived solutions change a lot along some straight lines. We call them "line dark soliton" solutions.

In Section 2 of this paper, the Lax pair for the nonlocal Davey-Stewartson I equation is reviewed and its symmetries are considered. Then the Darboux transformation is constructed and the explicit expressions of the new solutions are derived. In Section 3 and Section 4, the soliton solutions and "line dark soliton" solutions are constructed respectively. The globalness, boundedness and the asymptotic behaviors of those solutions are discussed.

\section{Lax pair and Darboux transformation}

Consider the $2 \times 2$ linear system

$$
\begin{aligned}
\Phi_{x}= & \tau J \Phi_{y}+\tau P \Phi=\tau\left(\begin{array}{cc}
1 & 0 \\
0 & -1
\end{array}\right) \Phi_{y}+\tau\left(\begin{array}{cc}
0 & -u \\
v & 0
\end{array}\right) \Phi, \\
\Phi_{t}= & -2 \mathrm{i} \tau^{2} J \Phi_{y y}-2 \mathrm{i} \tau^{2} P \Phi_{y}+\mathrm{i} Q \Phi \\
& =-2 \mathrm{i} \tau^{2}\left(\begin{array}{cc}
1 & 0 \\
0 & -1
\end{array}\right) \Phi_{y y}-2 \mathrm{i} \tau^{2}\left(\begin{array}{cc}
0 & -u \\
v & 0
\end{array}\right) \Phi_{y} \\
& +\mathrm{i} \tau\left(\begin{array}{cc}
-\tau u v-\tau w_{y}-w_{x} & u_{x}+\tau u_{y} \\
v_{x}-\tau v_{y} & \tau u v+\tau w_{y}-w_{x}
\end{array}\right) \Phi
\end{aligned}
$$

where $\tau= \pm 1, u, v, w$ are functions of $(x, y, t)$. The compatibility condition $\Phi_{x t}=\Phi_{t x}$ gives the evolution equation

$$
\begin{aligned}
& \mathrm{i} u_{t}=u_{x x}+\tau^{2} u_{y y}+2 \tau^{2} u^{2} v+2 \tau^{2} u w_{y} \\
& -\mathrm{i} v_{t}=v_{x x}+\tau^{2} v_{y y}+2 \tau^{2} u v^{2}+2 \tau^{2} v w_{y}, \\
& w_{x x}-\tau^{2} w_{y y}=2 \tau^{2}(u v)_{y} .
\end{aligned}
$$


When $\tau=1, v=\sigma \bar{u}^{*}(\sigma= \pm 1)$, (3) becomes the nonlocal Davey-Stewartson I equation (11). The Lax pair (3) becomes

$$
\begin{aligned}
\Phi_{x}= & U(\partial) \Phi \triangleq J \Phi_{y}+P \Phi=\left(\begin{array}{cc}
1 & 0 \\
0 & -1
\end{array}\right) \Phi_{y}+\left(\begin{array}{cc}
0 & -u \\
\sigma \bar{u}^{*} & 0
\end{array}\right) \Phi, \\
\Phi_{t}= & V(\partial) \Phi \triangleq-2 \mathrm{i} J \Phi_{y y}-2 \mathrm{i} P \Phi_{y}+\mathrm{i} Q \Phi \\
& =-2 \mathrm{i}\left(\begin{array}{cc}
1 & 0 \\
0 & -1
\end{array}\right) \Phi_{y y}-2 \mathrm{i}\left(\begin{array}{cc}
0 & -u \\
\sigma \bar{u}^{*} & 0
\end{array}\right) \Phi_{y} \\
& +\mathrm{i}\left(\begin{array}{cc}
-\sigma u \bar{u}^{*}-w_{y}-w_{x} & u_{x}+u_{y} \\
\sigma\left(\bar{u}^{*}\right)_{x}-\sigma\left(\bar{u}^{*}\right)_{y} & \sigma u \bar{u}^{*}+w_{y}-w_{x}
\end{array}\right) \Phi
\end{aligned}
$$

where $\partial=\frac{\partial}{\partial y}$. Here $U(\partial)$ implies that $U$ is a differential operator with respect to $y$.

The coefficients in the Lax pair (4) satisfies

$$
\bar{J}^{*}=-K J K^{-1}, \quad \bar{P}^{*}=-K P K^{-1}, \quad \bar{Q}^{*}=-K Q K^{-1}
$$

where $K=\left(\begin{array}{ll}0 & \sigma \\ 1 & 0\end{array}\right)$. Here $M^{*}$ refers to the complex conjugation (without transpose) of a matrix $M$. (15) implies

$$
\overline{U(\partial)^{*}}=-K U(\partial) K^{-1}, \quad \overline{V(\partial)^{*}}=K V(\partial) K^{-1} .
$$

Hence we have

Lemma 1 If $\Phi=\left(\begin{array}{c}\xi \\ \eta\end{array}\right)$ is a solution of (4), then so is $K \bar{\Phi}^{*}=\left(\begin{array}{c}\sigma \bar{\eta}^{*} \\ \bar{\xi}^{*}\end{array}\right)$.

By Lemma 1, take a solution $\left(\begin{array}{c}\xi \\ \eta\end{array}\right)$ of (4) and let $H=\left(\begin{array}{cc}\xi & \sigma \bar{\eta}^{*} \\ \eta & \bar{\xi}^{*}\end{array}\right)$, then $G(\partial)=\partial-S$ with $S=H_{y} H^{-1}$ gives a Darboux transformation. [18, 19] Written explicitly,

$$
S=\frac{1}{\xi \bar{\xi}^{*}-\sigma \eta \bar{\eta}^{*}}\left(\begin{array}{cc}
\bar{\xi}^{*} \xi_{y}-\sigma \eta\left(\bar{\eta}^{*}\right)_{y} & \sigma \xi\left(\bar{\eta}^{*}\right)_{y}-\sigma \bar{\eta}^{*} \xi_{y} \\
\bar{\xi}^{*} \eta_{y}-\eta\left(\bar{\xi}^{*}\right)_{y} & \xi\left(\bar{\xi}^{*}\right)_{y}-\sigma \bar{\eta}^{*} \eta_{y}
\end{array}\right)
$$

$G(\partial)$ also keeps the symmetries (5) invariant. After the action of $G(\partial),(u, w)$ is transformed to $(\widetilde{u}, \widetilde{w})$ by

$$
G(\partial) U(\partial)+G_{x}(\partial)=\widetilde{U}(\partial) G(\partial), \quad G(\partial) V(\partial)+G_{t}(\partial)=\widetilde{V}(\partial) G(\partial)
$$


That is,

$$
\begin{aligned}
& \widetilde{u}=u+2 \sigma \frac{\bar{\eta}^{*} \xi_{y}-\xi\left(\bar{\eta}^{*}\right)_{y}}{\bar{\xi}^{*} \xi-\sigma \bar{\eta}^{*} \eta}, \\
& \widetilde{w}=w+2 \frac{\left(\bar{\xi}^{*} \xi-\sigma \bar{\eta}^{*} \eta\right)_{y}}{\bar{\xi}^{*} \xi-\sigma \bar{\eta}^{*} \eta} .
\end{aligned}
$$

The Darboux transformation of degree $n$ is given by a matrix-valued differential operator

$$
G(\partial)=\partial^{n}+G_{1} \partial^{n-1}+\cdots+G_{n}
$$

of degree $n$ which is determined by

$$
G(\partial) H_{j}=0 \quad(j=1, \cdots, n)
$$

for $n$ matrix solutions $H_{j}(j=1, \cdots, n)$ of (2) . By comparing the coefficients of $\partial^{j}$ in (8)), the transformation of $(P, Q)$ is

$$
\widetilde{P}=P-\left[J, G_{1}\right], \quad \widetilde{Q}=Q+2\left[J, G_{2}\right]-2\left[J G_{1}-P, G_{1}\right]+4 J G_{1, y}-2 n P_{y} .
$$

Rewrite (11) as

$$
\partial^{n} H_{j}+G_{1} \partial^{n-1} H_{j}+\cdots+G_{n} H_{j}=0 \quad(j=1, \cdots, n),
$$

then

$$
\begin{aligned}
& \left(\begin{array}{llll}
G_{1} & G_{2} & \cdots & G_{n}
\end{array}\right)\left(\begin{array}{cccc}
\partial^{n-1} H_{1} & \partial^{n-1} H_{2} & \cdots & \partial^{n-1} H_{n} \\
\partial^{n-2} H_{1} & \partial^{n-2} H_{2} & \cdots & \partial^{n-2} H_{n} \\
\vdots & \vdots & & \vdots \\
H_{1} & H_{2} & \cdots & H_{n}
\end{array}\right) \\
& =\left(\begin{array}{llll}
-\partial^{n} H_{1} & -\partial^{n} H_{2} & \cdots & -\partial^{n} H_{n}
\end{array}\right) .
\end{aligned}
$$

Write $H_{j}=\left(\begin{array}{cc}h_{11}^{(j)} & h_{12}^{(j)} \\ h_{21}^{(j)} & h_{22}^{(j)}\end{array}\right)$. By reordering the rows and columns, we have

$$
\left(\begin{array}{llllll}
\left(G_{1}\right)_{11} & \cdots & \left(G_{n}\right)_{11} & \left(G_{1}\right)_{12} & \cdots & \left(G_{n}\right)_{12} \\
\left(G_{1}\right)_{21} & \cdots & \left(G_{n}\right)_{21} & \left(G_{1}\right)_{22} & \cdots & \left(G_{n}\right)_{22}
\end{array}\right) W=-R
$$

where $W=\left(W_{j k}\right)_{1 \leq j, k \leq 2}, R=\left(R_{j k}\right)_{1 \leq j, k \leq 2}$,

$$
W_{j k}=\left(\begin{array}{cccc}
\partial^{n-1} h_{j k}^{(1)} & \partial^{n-1} h_{j k}^{(2)} & \cdots & \partial^{n-1} h_{j k}^{(n)} \\
\partial^{n-2} h_{j k}^{(1)} & \partial^{n-2} h_{j k}^{(2)} & \cdots & \partial^{n-2} h_{j k}^{(n)} \\
\vdots & \vdots & & \vdots \\
h_{j k}^{(1)} & h_{j k}^{(2)} & \cdots & h_{j k}^{(n)}
\end{array}\right),
$$




$$
R_{j k}=\left(\begin{array}{llll}
\partial^{n} h_{j k}^{(1)} & \partial^{n} h_{j k}^{(2)} & \cdots & \partial^{n} h_{j k}^{(n)}
\end{array}\right) \quad(j, k=1,2) .
$$

Solving $G$ from (15), we get the new solution of the equation (11) from (12). Especially,

$$
\widetilde{u}=u+2\left(G_{1}\right)_{12} .
$$

\section{Soliton solutions}

\subsection{Single soliton solutions}

Let $u=0$, then $\Phi=\left(\begin{array}{l}\xi \\ \eta\end{array}\right)$ satisfies

$$
\Phi_{x}=\left(\begin{array}{cc}
1 & 0 \\
0 & -1
\end{array}\right) \Phi_{y}, \quad \Phi_{t}=-2 \mathrm{i}\left(\begin{array}{cc}
1 & 0 \\
0 & -1
\end{array}\right) \Phi_{y y} .
$$

Take a special solution

$$
\begin{aligned}
& \xi=\mathrm{e}^{\lambda x+\lambda y-2 \mathrm{i} \lambda^{2} t}+\mathrm{e}^{-\lambda^{*} x-\lambda^{*} y-2 \mathrm{i} \lambda^{* 2} t}, \\
& \eta=a \mathrm{e}^{\lambda x-\lambda y+2 \mathrm{i} \lambda^{2} t}+b \mathrm{e}^{-\lambda^{*} x+\lambda^{*} y+2 \mathrm{i} \lambda^{* 2} t},
\end{aligned}
$$

where $\lambda, a, b$ are complex constants. (9) gives the explicit solution

$$
\widetilde{u}=\frac{2 \sigma \lambda_{R}\left(a^{*}-b^{*}\right)}{D} \mathrm{e}^{2 \mathrm{i} \lambda_{I} y-4 \mathrm{i}\left(\lambda_{R}^{2}-\lambda_{I}^{2}\right) t}
$$

of (11) where

$$
\begin{aligned}
D= & \left(2-\sigma|a|^{2}-\sigma|b|^{2}\right) \cosh \left(2 \lambda_{R} y+8 \lambda_{R} \lambda_{I} t\right) \\
& +\sigma\left(|a|^{2}-|b|^{2}\right) \sinh \left(2 \lambda_{R} y+8 \lambda_{R} \lambda_{I} t\right) \\
& +2\left(1-\sigma \operatorname{Re}\left(a b^{*}\right)\right) \cosh \left(2 \lambda_{R} x\right)-2 \mathrm{i} \sigma \operatorname{Im}\left(a b^{*}\right) \sinh \left(2 \lambda_{R} x\right) .
\end{aligned}
$$

Here $z_{R}=\operatorname{Re} z$ and $z_{I}=\operatorname{Im} z$ for a complex number $z$. Since $\widetilde{w}$ is looked as an auxiliary function in (11), hereafter we will study mainly the behavior of $\widetilde{u}$.

Note that $\widetilde{u}$ is global if $|a|<1$ and $|b|<1$ since $\operatorname{Re} D>0$ in this case. Moreover, the peak moves in the velocity $\left(v_{x}, v_{y}\right)=\left(0,-4 \lambda_{I}\right)$.

Remark 1 The solution (21) may have singularities when the parameters are not chosen suitably, say, when $\sigma=-1, a=1, b=-2$, or $\sigma=1, a=2, b=1 / 4$.

Figure 1 shows a 1 soliton solution with parameters $\sigma=-1, t=20, \lambda=0.07-1.5 \mathrm{i}$, $a=0.2, b=0.1 \mathrm{i}$. 


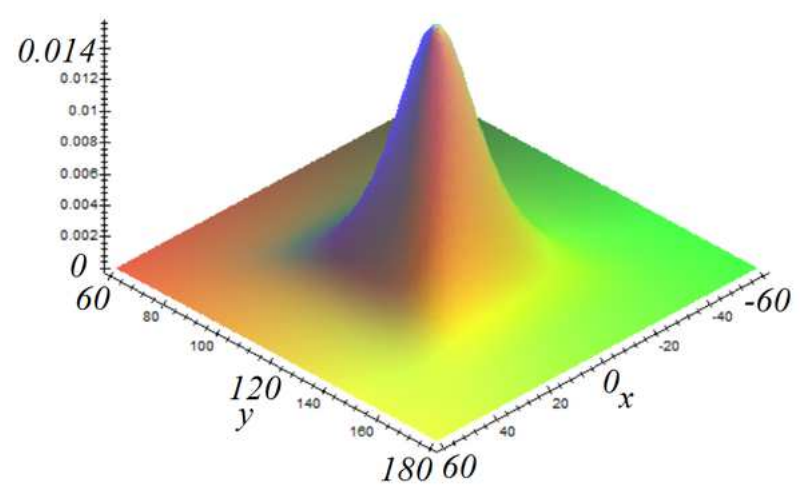

Figure 1: $|\widetilde{u}|$ of a 1 soliton solution.

\subsection{Multiple soliton solutions}

For an $n \times n$ matrix $M$, define $\|M\|=\sup _{x \in \mathbf{C}^{n},\|x\|=1}\|M x\|$ where $\|\cdot\|$ is the standard Hermitian norm in $\mathbf{C}^{n}$. The following facts hold obviously.

(i) $\|M N\| \leq\|M\|\|N\|$.

(ii) Each entry $M_{j k}$ of $M$ satisfies $\left|M_{j k}\right| \leq\|M\|$.

(iii) $|\operatorname{det} M| \leq\|M\|^{n}$.

(iv) If $\|M\|<1$, then $\left\|(I+M)^{-1}\right\| \leq(1-\|M\|)^{-1}$.

(v) If $\|M\|<1$, then $|\operatorname{det}(I+M)| \geq(1-\|M\|)^{n}$.

Now we construct explicit solutions according to (18). As in (20), take

$$
\begin{aligned}
& \xi_{k}=\mathrm{e}^{\lambda_{k}(x+y)-2 \mathrm{i} \lambda_{k}^{2} t}+\mathrm{e}^{-\lambda_{k}^{*}(x+y)-2 \mathrm{i} \lambda_{k}^{* 2} t}, \\
& \eta_{k}=a_{k} \mathrm{e}^{\lambda_{k}(x-y)+2 \mathrm{i} \lambda_{k}^{2} t}+b_{k} \mathrm{e}^{-\lambda_{k}^{*}(x-y)+2 \mathrm{i} \lambda_{k}^{* 2} t},
\end{aligned}
$$

then $W_{j k}$ 's in (16) and $R_{j k}$ 's in (17) are

$$
\begin{aligned}
& \left(W_{11}\right)_{j k}=\lambda_{k}^{n-j} e_{k+}+\left(-\lambda_{k}^{*}\right)^{n-j} e_{k+}^{*-1}, \quad\left(W_{12}\right)_{j k}=\sigma a_{k}^{*}\left(-\lambda_{k}^{*}\right)^{n-j} e_{k+}^{*-1}+\sigma b_{k}^{*} \lambda_{k}^{n-j} e_{k+}, \\
& \left(W_{21}\right)_{j k}=a_{k}\left(-\lambda_{k}\right)^{n-j} e_{k-}+b_{k}\left(\lambda_{k}^{*}\right)^{n-j} e_{k-}^{*-1}, \quad\left(W_{22}\right)_{j k}=\left(\lambda_{k}^{*}\right)^{n-j} e_{k-}^{*-1}+\left(-\lambda_{k}\right)^{n-j} e_{k-} \\
& \left(R_{11}\right)_{1 k}=\lambda_{k}^{n} e_{k+}+\left(-\lambda_{k}^{*}\right)^{n} e_{k+}^{*-1}, \quad\left(R_{12}\right)_{1 k}=\sigma a_{k}^{*}\left(-\lambda_{k}^{*}\right)^{n} e_{k+}^{*-1}+\sigma b_{k}^{*} \lambda_{k}^{n} e_{k+} \\
& \left(R_{21}\right)_{1 k}=a_{k}\left(-\lambda_{k}\right)^{n} e_{k-}+b_{k}\left(\lambda_{k}^{*}\right)^{n} e_{k-}^{*-1}, \quad\left(R_{22}\right)_{1 k}=\left(\lambda_{k}^{*}\right)^{n} e_{k-}^{*-1}+\left(-\lambda_{k}\right)^{n} e_{k-} \\
& (j, k=1, \cdots, n)
\end{aligned}
$$

where

$$
e_{k \pm}=\mathrm{e}^{\lambda_{k}(x \pm y) \mp 2 \mathrm{i} \lambda_{k}^{2} t} .
$$

However, temporarily, we assume $e_{k+}, e_{k-}(k=1, \cdots, n)$ are arbitrary complex numbers rather that (25) holds. 
Denote $L=\operatorname{diag}\left((-1)^{n-1},(-1)^{n-2}, \cdots,-1,1\right)$,

$$
\begin{gathered}
F=\left(\lambda_{k}^{n-j}\right)_{1 \leq j, k \leq n}, \quad f=\left(\lambda_{1}^{n}, \cdots, \lambda_{n}^{n}\right), \\
A=\operatorname{diag}\left(a_{1}, \cdots, a_{n}\right), \quad B=\operatorname{diag}\left(b_{1}, \cdots, b_{n}\right), \\
E_{ \pm}=\operatorname{diag}\left(e_{1 \pm}, \cdots, e_{n \pm}\right) .
\end{gathered}
$$

Then

$$
\begin{gathered}
W=\left(\begin{array}{cc}
F E_{+}+L F^{*} E_{+}^{*-1} & \sigma F B^{*} E_{+}+\sigma L F^{*} A^{*} E_{+}^{*-1} \\
L F A E_{-}+F^{*} B E_{-}^{*-1} & L F E_{-}+F^{*} E_{-}^{*-1}
\end{array}\right), \\
R=\left(\begin{array}{cc}
f E_{+}+(-1)^{n} f^{*} E_{+}^{*-1} & \sigma f B^{*} E_{+}+\sigma(-1)^{n} f^{*} A^{*} E_{+}^{*-1} \\
(-1)^{n} f A E_{-}+f^{*} B E_{-}^{*-1} & (-1)^{n} f E_{-}+f^{*} E_{-}^{*-1}
\end{array}\right) .
\end{gathered}
$$

By using the identity

$$
\left(\begin{array}{ll}
A & B \\
C & D
\end{array}\right)^{-1}=\left(\begin{array}{cc}
A^{-1}+A^{-1} B \Delta^{-1} C A^{-1} & -A^{-1} B \Delta^{-1} \\
-\Delta^{-1} C A^{-1} & \Delta^{-1}
\end{array}\right)
$$

for a block matrix where $\Delta=D-C A^{-1} B$, (15) gives

$$
\left(\left(G_{1}\right)_{12}, \cdots,\left(G_{n}\right)_{12}\right)=-\left(R W^{-1}\right)_{12}=-\left(R_{12}-R_{11} W_{11}^{-1} W_{12}\right) \stackrel{\circ}{W}^{-1}
$$

where

$$
\begin{aligned}
\stackrel{\circ}{W}= & W_{22}-W_{21} W_{11}^{-1} W_{12} \\
= & L\left(F E_{-}+L F^{*} E_{-}^{*-1}-\sigma\left(F A E_{-}+L F^{*} B E_{-}^{*-1}\right) .\right. \\
& \left.\cdot\left(F E_{+}+L F^{*} E_{+}^{*-1}\right)^{-1}\left(F B^{*} E_{+}+L F^{*} A^{*} E_{+}^{*-1}\right)\right) .
\end{aligned}
$$

Lemma 2 Suppose $a_{j}$ and $b_{j}$ are nonzero complex constants with $\left|a_{j}\right|<1,\left|b_{j}\right|<1(j=$ $1, \cdots, n), \kappa_{1}, \cdots, \kappa_{n}$ are nonzero real constants with $\left|\kappa_{j}\right| \neq\left|\kappa_{k}\right|(j, k=1, \cdots, n ; j \neq k)$, then there exist positive constants $\delta, C_{1}$ and $C_{2}$, which depend on $a_{j}$ 's, $b_{j}$ 's and $\kappa_{j}$ 's, such that $|\operatorname{det} W| \geq C_{1}$ and

$$
\left|\left(G_{1}\right)_{12}\right| \leq C_{2} \max _{1 \leq k \leq n} \frac{\left|e_{k+}\right|}{1+\left|e_{k+}\right|^{2}} \max _{1 \leq k \leq n} \frac{\left|e_{k-}\right|}{1+\left|e_{k-}\right|^{2}}
$$

hold whenever $\left|\lambda_{j}-\mathrm{i} \kappa_{j}\right|<\delta$ and $e_{j \pm} \in \mathbf{C}(j=1, \cdots, n)$.

Proof. Denote $F^{-1} L F^{*}=I+Z$, then $Z=0$ if $\lambda_{1}, \cdots, \lambda_{n}$ are all purely imaginary. From (29) and (33),

$$
\operatorname{det} W=\operatorname{det}\left(F E_{+}+L F^{*} E_{+}^{*-1}\right) \operatorname{det} \stackrel{\circ}{W},
$$




$$
\stackrel{\circ}{W}=L\left(F E_{-}+L F^{*} E_{-}^{*-1}\right)\left(I-\sigma \chi_{-} \chi_{+}\right)
$$

where

$$
\begin{gathered}
\chi_{+}=\left(F E_{+}+L F^{*} E_{+}^{*-1}\right)^{-1}\left(F B^{*} E_{+}+L F^{*} A^{*} E_{+}^{*-1}\right) \\
=\Xi_{1+} \Xi_{0+}^{-1}+\Xi_{0+}^{-1}\left(I+Z E_{+}^{*-1} \Xi_{0+}^{-1}\right)^{-1} Z E_{+}^{*-1}\left(A^{*}-\Xi_{1+} \Xi_{0+}^{-1}\right), \\
\chi_{-}=\left(F E_{-}+L F^{*} E_{-}^{*-1}\right)^{-1}\left(F A E_{-}+L F^{*} B E_{-}^{*-1}\right) \\
=\Xi_{1-} \Xi_{0-}^{-1}+\Xi_{0-}^{-1}\left(I+Z E_{-}^{*-1} \Xi_{0-}^{-1}\right)^{-1} Z E_{-}^{*-1}\left(B-\Xi_{1-} \Xi_{0-}^{-1}\right), \\
\Xi_{0 \pm}=E_{ \pm}+E_{ \pm}^{*-1}, \quad \Xi_{1-}=A E_{-}+B E_{-}^{*-1}, \quad \Xi_{1+}=B^{*} E_{+}+A^{*} E_{+}^{*-1} .
\end{gathered}
$$

Let $c_{0}=\max _{1 \leq k \leq n}\left\{\left|a_{k}\right|,\left|b_{k}\right|\right\}<1$. Suppose $\|Z\|<\frac{1-c_{0}}{2}$, then we have the following estimates.

$$
\begin{gathered}
\|A\| \leq c_{0}<1, \quad\|B\| \leq c_{0}<1, \\
\left\|E_{ \pm} \Xi_{0 \pm}^{-1}\right\| \leq 1, \quad\left\|E_{ \pm}^{*-1} \Xi_{0 \pm}^{-1}\right\| \leq 1, \\
\left\|\Xi_{0 \pm}\right\| \geq 2, \quad\left\|\Xi_{0 \pm}^{-1}\right\| \leq \frac{1}{2}, \quad\left\|\Xi_{1 \pm} \Xi_{0 \pm}^{-1}\right\| \leq c_{0}<1, \\
\left\|E_{+}^{*-1}\left(\Xi_{1+} \Xi_{0+}^{-1}-A^{*}\right)\right\|=\max _{1 \leq k \leq n} \frac{\left|a_{k}-b_{k}\right|\left|e_{k+}\right|}{1+\left|e_{k+}\right|^{2}} \leq 1, \\
\left\|E_{-}^{*-1}\left(\Xi_{1-} \Xi_{0-}^{-1}-B\right)\right\|=\max _{1 \leq k \leq n} \frac{\left|a_{k}-b_{k}\right|\left|e_{k-}\right|}{1+\left|e_{k-}\right|^{2}} \leq 1, \\
\left\|\left(I+Z E_{ \pm}^{*-1} \Xi_{0 \pm}^{-1}\right)^{-1}\right\| \leq\left\|\left(1-\|Z\|\left|\| E_{ \pm}^{*-1} \Xi_{0 \pm}^{-1}\right| \mid\right)^{-1}\right\| \leq(1-\|Z\|)^{-1} \leq 2 .
\end{gathered}
$$

Hence $\left\|\chi_{ \pm}-\Xi_{1 \pm} \Xi_{0 \pm}^{-1}\right\| \leq\|Z\|$,

$$
\left\|\chi_{ \pm}\right\| \leq c_{0}+\|Z\| \leq \frac{1+c_{0}}{2}<1 .
$$

Denote

$$
\pi_{0}=\left.|\operatorname{det} F|\right|_{\substack{\lambda_{j}=\mathrm{i} \kappa_{j} \\ j=1, \cdots, n}}, \quad \pi_{1}=\left\|\left.F^{-1}||\right|_{\substack{\lambda_{j}=\mathrm{i} \kappa_{j} \\ j=1, \cdots, n}}, \quad \pi_{2}=\right\| f \|\left.\right|_{\substack{\lambda_{j}=\mathrm{i} \kappa_{j} \\ j=1, \cdots, n}}
$$

Clearly, $\pi_{0}, \pi_{1}, \pi_{2}$ are all positive since $\left.\operatorname{det} F\right|_{\substack{\lambda_{j}=\mathrm{i} \kappa_{j} \\ j=1, \ldots, n}}$ is a Vandermonde determinant. By the continuity, there exists $\delta>0$ such that $\frac{\pi_{0}}{2} \leq|\operatorname{det} F| \leq 2 \pi_{0},\left\|F^{-1}\right\| \leq 2 \pi_{1},\|f\| \leq 2 \pi_{2}$, and ||$F^{-1} L F^{*}-I\|=\| Z \|<\frac{1-c_{0}}{2}$ whenever $\left|\lambda_{j}-\mathrm{i} \kappa_{j}\right|<\delta$. (35) and (36) lead to

$$
\begin{aligned}
& |\operatorname{det} W|=|\operatorname{det} F|^{2}\left|\operatorname{det} \Xi_{0+}\right|\left|\operatorname{det}\left(I+Z E_{+}^{*-1} \Xi_{0+}^{-1}\right)\right| \cdot \\
& \quad \cdot\left|\operatorname{det} \Xi_{0-}\right|\left|\operatorname{det}\left(I+Z E_{-}^{*-1} \Xi_{0-}^{-1}\right)\right|\left|\operatorname{det}\left(1-\sigma \chi_{-} \chi_{+}\right)\right| \\
& \quad \geq \pi_{0}^{2}(1-|| Z||)^{2 n}\left(1-|| \chi_{+}|||| \chi_{-}||\right)^{n} \geq \pi_{0}^{2}\left(\frac{1+c_{0}}{2}\right)^{2 n}\left(1-\left(\frac{1+c_{0}}{2}\right)^{2}\right)^{n}
\end{aligned}
$$


which is a uniform positive lower bound for any $e_{j \pm} \in \mathbf{C}(j=1, \cdots, n)$ when $\left|\lambda_{j}-\mathrm{i} \kappa_{j}\right|<\delta$.

By (29), (30), (32) and (33),

$$
\begin{aligned}
\left(\left(G_{1}\right)_{12},\right. & \left.\left(G_{2}\right)_{12}, \cdots,\left(G_{n}\right)_{12}\right)=-\left(R_{12}-R_{11} W_{11}^{-1} W_{12}\right) \stackrel{\circ}{W} \\
= & -\sigma f E_{+} \Xi_{0+}^{-1}\left(I+Z E_{+}^{*-1} \Xi_{0+}^{-1}\right)^{-1}(I+Z)\left(B^{*}-A^{*}\right) E_{+}^{*-1} \stackrel{\circ}{W}^{-1} \\
& -\sigma(-1)^{n} f^{*} E_{+}^{*-1} \Xi_{0+}^{-1}\left(I+Z E_{+}^{*-1} \Xi_{0+}^{-1}\right)^{-1}\left(A^{*}-B^{*}\right) E_{+} \stackrel{\circ}{W}^{-1} \\
= & -\sigma\left(f-(-1)^{n} f^{*}+\left(f E_{+} \Xi_{0+}^{-1}+(-1)^{n} f^{*} E_{+}^{*-1} \Xi_{0+}^{-1}\right)\left(I+Z E_{+}^{*-1} \Xi_{0+}^{-1}\right)^{-1} Z\right) . \\
& \cdot\left(B^{*}-A^{*}\right) E_{+} E_{+}^{*-1} \Xi_{0+}^{-1}\left(I-\sigma \chi_{-} \chi_{+}\right)^{-1} \Xi_{0-}^{-1}\left(I+Z E_{-}^{*-1} \Xi_{0-}^{-1}\right)^{-1} F^{-1} L^{-1} .
\end{aligned}
$$

Here we have used $I+Z=\left(I+Z E_{+}^{*-1} \Xi_{0+}^{-1}\right)+Z E_{+} \Xi_{0+}^{-1}$. Hence, by using (39)-(44),

$$
\begin{aligned}
& \left|\left(G_{1}\right)_{12}\right| \leq 8\left(\|f\|+\| f^{*}||\right)|| F^{-1}||||\left(I-\sigma \chi_{-} \chi_{+}\right)^{-1}||\left\|\Xi_{0+}^{-1}||\right\| \Xi_{0-}^{-1}|| \\
& \quad \leq 64 \pi_{1} \pi_{2}\left(1-\left(\frac{1+c_{0}}{2}\right)^{2}\right)^{-1} \max _{1 \leq k \leq n} \frac{\left|e_{k+}\right|}{1+\left|e_{k+}\right|^{2}} \max _{1 \leq k \leq n} \frac{\left|e_{k-}\right|}{1+\left|e_{k-}\right|^{2}}
\end{aligned}
$$

The lemma is proved.

Now we consider the solutions of the nonlocal Davey-Stewartson I equation. That is, we consider the case where $e_{j \pm}$ 's are taken as (25).

Theorem 1 Suppose $a_{j}$ and $b_{j}$ are nonzero complex constants with $\left|a_{j}\right|<1,\left|b_{j}\right|<1(j=$ $1, \cdots, n), \kappa_{1}, \cdots, \kappa_{n}$ are nonzero real constants with $\left|\kappa_{j}\right| \neq\left|\kappa_{k}\right|(j, k=1, \cdots, n ; j \neq k)$, then there exists a positive constant $\delta$ such that the following results hold for the derived solution $\widetilde{u}=2\left(G_{1}\right)_{12}$ of the nonlocal Davey-Stewartson I equation when $\operatorname{Re} \lambda_{j} \neq 0$ and $\left|\lambda_{j}-\mathrm{i} \kappa_{j}\right|<\delta(j=1, \cdots, n)$.

(i) $\widetilde{u}$ is defined globally for $(x, y, t) \in \mathbf{R}^{3}$.

(ii) For fixed $t, \widetilde{u}$ tends to zero exponentially as $(x, y) \rightarrow 0$.

(iii) Let $y=\widetilde{y}+v t$ and keep $(x, \widetilde{y})$ bounded, then $\widetilde{u} \rightarrow 0$ as $t \rightarrow \infty$ if $v \neq-4 \lambda_{k I}$ for all $k$.

Proof. We have known that $\widetilde{u}$ is a solution of the nonlocal Davey-Stewartson equation in Section 2.

(i) According to Lemma 2, $|\operatorname{det} W|$ has a uniform positive lower bound. Hence $\widetilde{u}$ is defined globally.

(ii) When $x \geq 0$ and $y \geq 0$,

$$
\begin{aligned}
& \left|e_{k+}\right| \geq \mathrm{e}^{\lambda_{k R} \sqrt{x^{2}+y^{2}}+4 \lambda_{k R} \lambda_{k I} t} \text { if } \lambda_{k R}>0, \\
& \left|e_{k+}\right| \leq \mathrm{e}^{-\left|\lambda_{k R}\right| \sqrt{x^{2}+y^{2}}+4 \lambda_{k R} \lambda_{k I} t} \text { if } \lambda_{k R}<0 .
\end{aligned}
$$

Hence $\max _{1 \leq k \leq n} \frac{\left|e_{k+}\right|}{1+\left|e_{k+}\right|^{2}}$ tends to zero exponentially when $x \geq 0, y \geq 0$ and $(x, y) \rightarrow \infty$. 


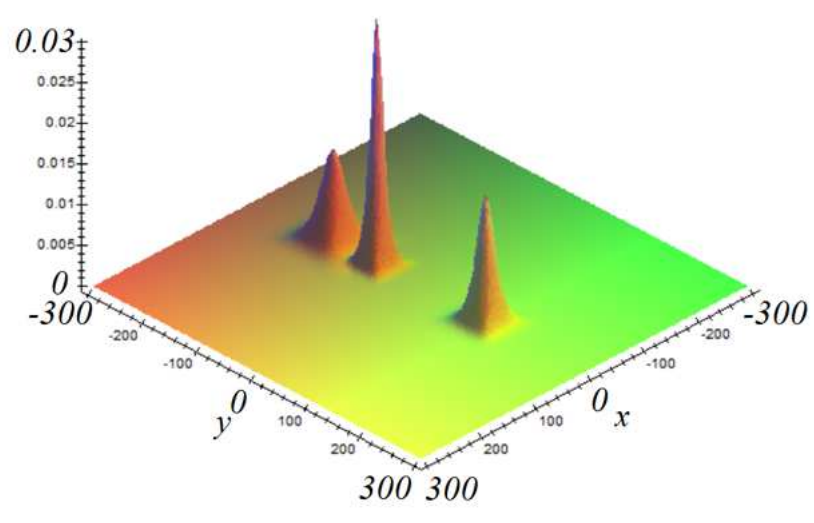

Figure 2: $|\widetilde{u}|$ of a 3 soliton solution.

Likewise, we have

$$
\begin{aligned}
& \left|e_{k-}\right| \leq \mathrm{e}^{-\lambda_{k R} \sqrt{x^{2}+y^{2}}-4 \lambda_{k R} \lambda_{k I} t} \text { if } x \leq 0, y \geq 0, \lambda_{k R}>0, \\
& \left|e_{k-}\right| \geq \mathrm{e}^{\left|\lambda_{k R}\right| \sqrt{x^{2}+y^{2}}-4 \lambda_{k R} \lambda_{k I} t} \text { if } x \leq 0, y \geq 0, \lambda_{k R}<0, \\
& \left|e_{k+}\right| \leq \mathrm{e}^{-\lambda_{k R} \sqrt{x^{2}+y^{2}}+4 \lambda_{k R} \lambda_{k I} t} \text { if } x \leq 0, y \leq 0, \lambda_{k R}>0, \\
& \left|e_{k+}\right| \geq \mathrm{e}^{\left|\lambda_{k R}\right| \sqrt{x^{2}+y^{2}}+4 \lambda_{k R} \lambda_{k I} t} \text { if } x \leq 0, y \leq 0, \lambda_{k R}<0, \\
& \left|e_{k-}\right| \geq \mathrm{e}^{\lambda_{k R} \sqrt{x^{2}+y^{2}}-4 \lambda_{k R} \lambda_{k I} t} \text { if } x \geq 0, y \leq 0, \lambda_{k R}>0, \\
& \left|e_{k-}\right| \leq \mathrm{e}^{-\left|\lambda_{k R}\right| \sqrt{x^{2}+y^{2}}-4 \lambda_{k R} \lambda_{k I} t} \text { if } x \geq 0, y \leq 0, \lambda_{k R}<0 .
\end{aligned}
$$

Lemma 2 implies that $\widetilde{u} \rightarrow 0$ exponentially as $(x, y) \rightarrow \infty$.

(iii)

$$
\left|e_{k \pm}\right|=\mathrm{e}^{\lambda_{k R}(x \pm \widetilde{y}) \pm \lambda_{k R}\left(v+4 \lambda_{k I}\right) t}
$$

If $v \neq-4 \lambda_{k I}$ for all $k=1, \cdots, n$, then either $e_{k+} \rightarrow 0$ or $e_{k+} \rightarrow \infty$ for all $k=1, \cdots, n$ when $t \rightarrow \infty$. Lemma 2 implies that $\widetilde{u} \rightarrow 0$ when $t \rightarrow \infty$. The theorem is proved.

A 3 soliton solution is shown in Figure 2 where the parameters are $\sigma=-1, t=20$, $\lambda_{1}=0.07-1.5 \mathrm{i}, \lambda_{2}=0.05+2 \mathrm{i}, \lambda_{3}=0.1+\mathrm{i}, a_{1}=0.2, a_{2}=0.1 \mathrm{i}, a_{3}=0.1, b_{1}=0.1 \mathrm{i}$, $b_{2}=-0.2, b_{3}=-0.2$. The figure of the solution appears similarly if $\sigma$ is changed to +1 , although it is not shown here. 


\section{4 "Line dark soliton" solutions}

\subsection{Single "line dark soliton" solutions}

Now we take

$$
u=\rho \mathrm{e}^{-2 \mathrm{i} \sigma|\rho|^{2} t}, \quad w=0
$$

as a solution of (1) where $\rho$ is a complex constant. The Lax pair (4) has a solution

$$
\left(\begin{array}{c}
\mathrm{e}^{\alpha(\lambda) x+\beta(\lambda) y+\gamma(\lambda) t} \\
\frac{\lambda}{\rho} \mathrm{e}^{\alpha(\lambda) x+\beta(\lambda) y+\left(\gamma(\lambda)+2 \mathrm{i} \sigma|\rho|^{2}\right) t}
\end{array}\right)
$$

where

$$
\begin{aligned}
& \alpha(\lambda)=\frac{1}{2}\left(\frac{\sigma|\rho|^{2}}{\lambda}-\lambda\right), \quad \beta(\lambda)=\frac{1}{2}\left(\frac{\sigma|\rho|^{2}}{\lambda}+\lambda\right) \\
& \gamma(\lambda)=\mathrm{i}\left(\alpha(\lambda)^{2}-2 \alpha(\lambda) \beta(\lambda)-\beta(\lambda)^{2}\right)=\mathrm{i} \lambda^{2}-\frac{\mathrm{i}}{2}\left(\frac{\sigma|\rho|^{2}}{\lambda}+\lambda\right)^{2},
\end{aligned}
$$

$\lambda$ is a complex constant. Note that $\alpha\left(-\lambda^{*}\right)=-(\alpha(\lambda))^{*}, \beta\left(-\lambda^{*}\right)=-(\beta(\lambda))^{*}, \gamma\left(-\lambda^{*}\right)=$ $-(\gamma(\lambda))^{*}$

$$
\begin{aligned}
& \text { Now take } \Phi=\left(\begin{array}{c}
\xi \\
\eta
\end{array}\right) \text { where } \\
& \qquad \begin{array}{l}
\xi=\mathrm{e}^{\alpha x+\beta y+\gamma t}+\mathrm{e}^{-\alpha^{*} x-\beta^{*} y-\gamma^{*} t}, \\
\eta=\frac{\lambda}{\rho} \mathrm{e}^{\alpha x+\beta y+\left(\gamma+2 \mathrm{i} \sigma|\rho|^{2}\right) t}-\frac{\lambda^{*}}{\rho} \mathrm{e}^{-\alpha^{*} x-\beta^{*} y-\left(\gamma^{*}-2 \mathrm{i} \sigma|\rho|^{2}\right) t} .
\end{array}
\end{aligned}
$$

Here $\alpha=\alpha(\lambda), \beta=\beta(\lambda), \gamma=\gamma(\lambda)$. This $\Phi$ is a linear combination of the solutions of form (53). Then (9) gives the new solution

$$
\widetilde{u}=\rho \mathrm{e}^{-2 \mathrm{i} \sigma|\rho|^{2} t} \frac{\frac{\lambda^{*}}{\lambda} c_{1} \mathrm{e}^{2 \beta_{R} y+2 \gamma_{R} t}+\frac{\lambda}{\lambda^{*}} c_{1} \mathrm{e}^{-2 \beta_{R} y-2 \gamma_{R} t}-c_{2} \mathrm{e}^{2 \alpha_{R} x}-c_{2}^{*} \mathrm{e}^{-2 \alpha_{R} x}}{c_{1}\left(\mathrm{e}^{2 \beta_{R} y+2 \gamma_{R} t}+\mathrm{e}^{-2 \beta_{R} y-2 \gamma_{R} t}\right)+c_{2} \mathrm{e}^{2 \alpha_{R} x}+c_{2}^{*} \mathrm{e}^{-2 \alpha_{R} x}}
$$

of the nonlocal Davey-Stewartson I equation where

$$
c_{1}=1-\sigma \frac{|\lambda|^{2}}{|\rho|^{2}}, \quad c_{2}=1+\sigma \frac{\lambda^{2}}{|\rho|^{2}}
$$

This solution is smooth for all $(x, y, t) \in \mathbf{R}^{3}$ if $|\lambda|<|\rho|$.

Especially, if $\lambda$ is real, then $\gamma_{R}=0$, so we get a standing wave solution.

Figure 3 shows a 1 "line dark soliton" solution with parameters $\sigma=-1, t=10, \rho=1$, $\lambda=0.3+0.1 \mathrm{i}$. The figure on the right describes the same solution but is upside down. 

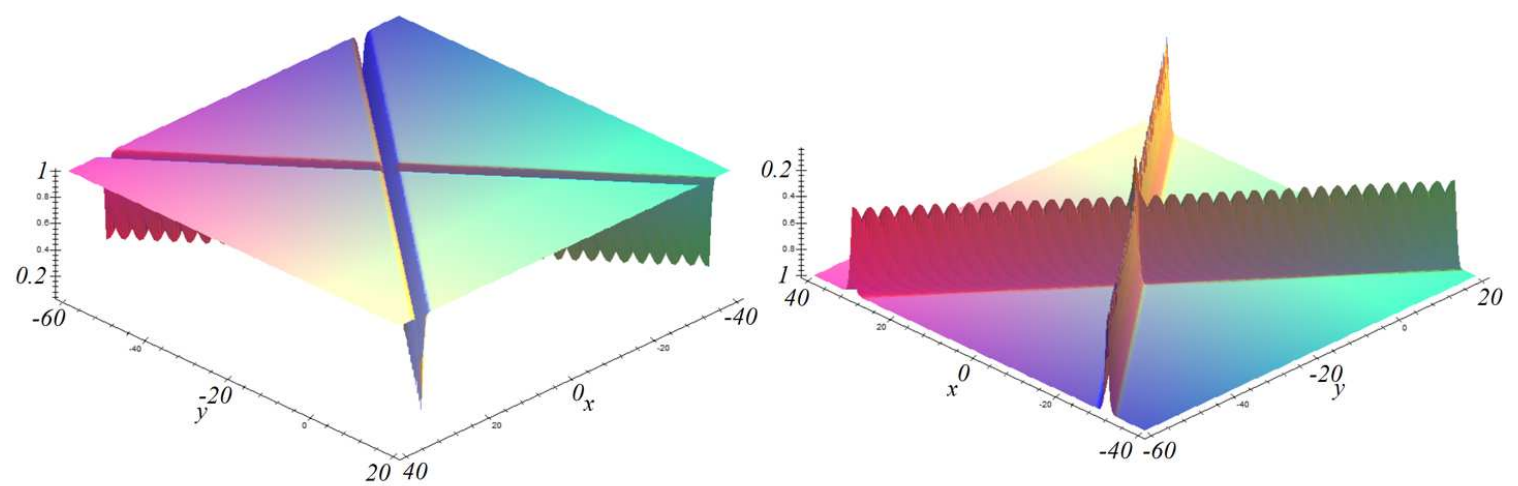

Figure 3: $|\widetilde{u}|$ of a 1 "line dark soliton" solution.

\subsection{Multiple "line dark soliton" solutions}

Now we take $n$ solutions

$$
\begin{aligned}
& \xi_{k}=\mathrm{e}^{\alpha_{k} x+\beta_{k} y+\gamma_{k} t}+\mathrm{e}^{-\alpha_{k}^{*} x-\beta_{k}^{*} y-\gamma_{k}^{*} t}, \\
& \eta_{k}=\frac{\lambda_{k}}{\rho} \mathrm{e}^{\alpha_{k} x+\beta_{k} y+\left(\gamma_{k}+2 \mathrm{i} \sigma|\rho|^{2}\right) t}-\frac{\lambda_{k}^{*}}{\rho} \mathrm{e}^{-\alpha_{k}^{*} x-\beta_{k}^{*} y-\left(\gamma_{k}^{*}-2 \mathrm{i} \sigma|\rho|^{2}\right) t}
\end{aligned}
$$

of form (55) to get multiple "line dark soliton" solutions where

$$
\begin{aligned}
& \alpha_{k}=\frac{1}{2}\left(\frac{\sigma|\rho|^{2}}{\lambda_{k}}-\lambda_{k}\right), \quad \beta_{k}=\frac{1}{2}\left(\frac{\sigma|\rho|^{2}}{\lambda_{k}}+\lambda_{k}\right), \\
& \gamma_{k}=\mathrm{i}\left(\alpha_{k}^{2}-2 \alpha_{k} \beta_{k}-\beta_{k}^{2}\right) .
\end{aligned}
$$

Similar to (29) and (30), $W$ and $R$ in (15) are

$$
\begin{gathered}
W=\left(\begin{array}{cc}
F E_{+}+L F^{*} E_{+}^{*-1} & -\sigma \rho^{*-1} \mathrm{e}^{-\mathrm{i} \phi}\left(L F \Lambda E_{-}-F^{*} \Lambda^{*} E_{-}^{*-1}\right) \\
\rho^{-1} \mathrm{e}^{\mathrm{i} \phi}\left(F \Lambda E_{+}-L F^{*} \Lambda^{*} E_{+}^{*-1}\right) & L F E_{-}+F^{*} E_{-}^{*-1}
\end{array}\right), \\
R=\left(\begin{array}{cc}
f E_{+}+(-1)^{n} f^{*} E_{+}^{*-1} & -\sigma \rho^{*-1} \mathrm{e}^{-\mathrm{i} \phi}\left((-1)^{n} f \Lambda E_{-}-f^{*} \Lambda^{*} E_{-}^{*-1}\right) \\
\rho^{-1} \mathrm{e}^{\mathrm{i} \phi}\left(f \Lambda E_{+}-(-1)^{n} f^{*} \Lambda^{*} E_{+}^{*-1}\right) & (-1)^{n} f E_{-}+f^{*} E_{-}^{*-1}
\end{array}\right),
\end{gathered}
$$

where

$$
\begin{aligned}
& E_{ \pm}=\operatorname{diag}\left(e_{k \pm}\right)_{k=1, \cdots, n}, \quad F=\left(\beta_{k}^{n-j}\right)_{1 \leq j, k \leq n}, \quad f=\left(\beta_{1}^{n}, \cdots, \beta_{n}^{n}\right), \\
& \alpha_{k}=\frac{1}{2}\left(\frac{\sigma|\rho|^{2}}{\lambda_{k}}-\lambda_{k}\right), \quad \beta_{k}=\frac{1}{2}\left(\frac{\sigma|\rho|^{2}}{\lambda_{k}}+\lambda_{k}\right), \\
& \gamma_{k}=\mathrm{i}\left(\alpha_{k}^{2}-2 \alpha_{k} \beta_{k}-\beta_{k}^{2}\right),
\end{aligned}
$$

$\Lambda=\operatorname{diag}\left(\lambda_{k}\right)_{1 \leq k \leq n}, \phi=2 \sigma|\rho|^{2} t$. Moreover, $e_{k \pm}=\mathrm{e}^{\alpha_{k} x \pm \beta_{k} y \pm \gamma_{k} t}$. However, as in the soliton case, we suppose temporarily that $e_{k \pm}$ 's are arbitrary complex numbers. 
Lemma 3 Suppose $\kappa_{1}, \cdots, \kappa_{n}$ are distinct nonzero real numbers, then there exist positive constants $\rho_{0}, \delta, C_{1}$ and $C_{2}$, which depend on $\kappa_{j}$ 's, such that $|\operatorname{det} W| \geq C_{1}$ and $\left|\left(G_{1}\right)_{12}\right| \leq$ $C_{2}$ hold whenever $|\rho|>\rho_{0},\left|\lambda_{j}-\mathrm{i} \kappa_{j}\right|<\delta$ and $e_{j \pm} \in \mathbf{C}(j=1, \cdots, n)$.

Proof. Denote $F^{-1} L F^{*}=I+Z$, then $Z=0$ if $\lambda_{1}, \cdots, \lambda_{n}$ are all purely imaginary. Hence $\|Z\|$ is small enough if $\left|\lambda_{1}-\mathrm{i} \kappa_{1}\right|, \cdots,\left|\lambda_{n}-\mathrm{i} \kappa_{n}\right|$ are all small enough.

Let $c_{0}=\max _{1 \leq k \leq n}\left|\kappa_{k}\right|, \pi_{3}=\left.|\operatorname{det} F|\right|_{\substack{\lambda_{k}=\mathrm{i} \kappa_{k} \\ k=1, \cdots, n}}, \pi_{4}=\| F^{-1} L F||_{\substack{\lambda_{k}=\mathrm{i} \kappa_{k} \\ k=1, \cdots, n}}$. Then there exists $\delta$ with $0<\delta<c_{0}$ such that $\|Z\| \leq \frac{1}{2},|\operatorname{det} F| \geq \frac{\pi_{3}}{2},\left\|F^{-1} L F\right\| \leq 2 \pi_{4}$ if $\left|\lambda_{k}-\mathrm{i} \kappa_{k}\right|<\delta$ $(k=1, \cdots, n)$. In this case, $\left|\lambda_{k}\right|<c_{0}+\delta<2 c_{0}$.

From (60),

$$
\operatorname{det} W=\operatorname{det}\left(F E_{+}+L F^{*} E_{+}^{*-1}\right) \operatorname{det} \stackrel{\circ}{W}
$$

where

$$
\begin{aligned}
& \stackrel{\circ}{W}= L F E_{-}+F^{*} E_{-}^{*-1}+\sigma|\rho|^{-2}\left(F \Lambda E_{+}-L F^{*} \Lambda^{*} E_{+}^{*-1}\right) . \\
& \cdot\left(F E_{+}+L F^{*} E_{+}^{*-1}\right)^{-1} L\left(F \Lambda E_{-}-L F^{*} \Lambda^{*} E_{-}^{*-1}\right) \\
&=\left(1+\sigma|\rho|^{-2} F \chi_{+} F^{-1} L F \chi_{-} F^{-1} L^{-1}\right) L F\left(I+Z E_{-}^{*-1} \Xi_{0-}^{-1}\right) \Xi_{0-} \\
& \chi_{ \pm}=F^{-1}\left(F \Lambda E_{ \pm}-L F^{*} \Lambda^{*} E_{ \pm}^{*-1}\right)\left(F E_{ \pm}+L F^{*} E_{ \pm}^{*-1}\right)^{-1} F \\
&=\Xi_{1 \pm} \Xi_{0 \pm}^{-1}-\left(Z \Lambda^{*}+\Xi_{1 \pm} \Xi_{0 \pm}^{-1} Z\right) E_{ \pm}^{*-1} \Xi_{0 \pm}^{-1}\left(I+Z E_{ \pm}^{*-1} \Xi_{0 \pm}^{-1}\right)^{-1} \\
& \Xi_{0 \pm}=E_{ \pm}+E_{ \pm}^{*-1}, \quad \Xi_{1 \pm}=\Lambda E_{ \pm}-\Lambda^{*} E_{ \pm}^{*-1} .
\end{aligned}
$$

We have the following estimates:

$$
\begin{gathered}
\left\|E_{ \pm} \Xi_{0 \pm}^{-1}\right\| \leq 1, \quad\left\|E_{ \pm}^{*-1} \Xi_{0 \pm}^{-1}\right\| \leq 1, \quad\left\|\Xi_{1 \pm} \Xi_{0 \pm}^{-1}\right\| \leq 2 c_{0} \\
\left\|\Xi_{0 \pm}\right\| \geq 2, \quad\left\|\Xi_{0 \pm}^{-1}\right\| \leq \frac{1}{2}, \quad\left|\operatorname{det} \Xi_{0 \pm}\right| \geq 2^{n} \\
\left\|\left(I+Z E_{ \pm}^{*-1} \Xi_{0 \pm}^{-1}\right)^{-1}\right\| \leq(1-\|Z\|)^{-1} \leq 2, \quad\left|\operatorname{det}\left(I+Z E_{ \pm}^{*-1} \Xi_{0 \pm}^{-1}\right)\right| \geq(1-\|Z\|)^{n} \geq \frac{1}{2^{n}} .
\end{gathered}
$$

Hence (65) implies

$$
\begin{gathered}
\left\|\chi_{ \pm}\right\| \leq 2 c_{0}+8 c_{0}\|Z\| \leq 6 c_{0} \\
\left\|\chi_{+} F^{-1} L F \chi_{-} F^{-1} L^{-1} F\right\| \leq\left\|F^{-1} L F\right\|^{2}\left\|\chi_{+}\right\|\left\|\chi_{-}\right\| \leq 144 c_{0}^{2} \pi_{4}^{2} .
\end{gathered}
$$

By (63) and (64),

$$
\begin{aligned}
& |\operatorname{det} W|=|\operatorname{det} F|^{2}\left|\operatorname{det} \Xi_{0+}\right|\left|\operatorname{det} \Xi_{0-}\right|\left|\operatorname{det}\left(I+Z E_{+}^{*-1} \Xi_{0+}^{-1}\right)\right| \cdot \\
& \quad \cdot\left|\operatorname{det}\left(I+Z E_{-}^{*-1} \Xi_{0-}^{-1}\right)\right|\left|\operatorname{det}\left(I+\sigma|\rho|^{-2} \chi_{+} F^{-1} L F \chi_{-} F^{-1} L^{-1} F\right)\right| \\
& \geq \frac{\pi_{3}^{2}}{4}\left(1-144 c_{0}^{2} \pi_{4}^{2}|\rho|^{-2}\right)>0
\end{aligned}
$$


if $|\rho|>12 c_{0} \pi_{4}$. Therefore, $|\operatorname{det} W|$ has a uniform positive lower bound if $|\rho|>12 c_{0} \pi_{4}$ and $\left|\lambda_{k}-\mathrm{i} \kappa_{k}\right|<\delta(k=1, \cdots, n)$.

By (15), (60), (61), (63) and (64),

$$
\begin{aligned}
& \left(\left(G_{1}\right)_{12},\left(G_{2}\right)_{12}, \cdots,\left(G_{n}\right)_{12}\right)=-\left(R_{12}-R_{11} W_{11}^{-1} W_{12}\right) \stackrel{\circ}{W} \\
& =\sigma \rho^{*-1} \mathrm{e}^{-\mathrm{i} \phi}(-1)^{n}\left(f \Lambda E_{-}-(-1)^{n} f^{*} \Lambda^{*} E_{-}^{*-1}-\left(f E_{+}+(-1)^{n} f^{*} E_{+}^{*-1}\right) .\right. \\
& \left.\quad \cdot(-1)^{n}\left(F E_{+}+L F^{*} E_{+}^{*-1}\right)^{-1} L\left(F \Lambda E_{-}-L F^{*} \Lambda^{*} E_{-}^{*-1}\right)\right) \stackrel{\circ}{W}^{-1} \\
& =\sigma \rho^{*-1} \mathrm{e}^{-\mathrm{i} \phi}(-1)^{n}\left(f \Lambda E_{-} \Xi_{0-}^{-1}-(-1)^{n} f^{*} \Lambda^{*} E_{-}^{*-1} \Xi_{0-}^{-1}\right. \\
& \quad-(-1)^{n}\left(f E_{+} \Xi_{0+}^{-1}+(-1)^{n} f^{*} E_{+}^{*-1} \Xi_{0+}^{-1}\right)\left(I+Z E_{+}^{*-1} \Xi_{0+}^{-1}\right)^{-1} F^{-1} L F . \\
& \left.\quad \cdot\left(\Xi_{1-} \Xi_{0-}^{-1}-Z \Lambda^{*} E_{-}^{*-1} \Xi_{0-}^{-1}\right)\right)\left(I+Z E_{-}^{*-1} \Xi_{0-}^{-1}\right)^{-1} F^{-1} L^{-1} . \\
& \quad \cdot\left(1+\sigma|\rho|^{-2} F \chi_{+} F^{-1} L F \chi_{-} F^{-1} L^{-1}\right)^{-1} .
\end{aligned}
$$

$\left(G_{1}\right)_{12}$ is bounded when $|\rho|>12 c_{0} \pi_{4}$ and $\left|\lambda_{k}-\mathrm{i} \kappa_{k}\right|<\delta(k=1, \cdots, n)$ because of the estimates (67)-(72). The lemma is proved.

Now we have the following theorem for the multiply "line dark soliton" solution.

Theorem 2 Suppose $\kappa_{1}, \cdots, \kappa_{n}$ are distinct nonzero real numbers, then there exist positive constants $\rho_{0}$ and $\delta$ such that the following results hold for the derived solution $\widetilde{u}=$ $u+2\left(G_{1}\right)_{12}$ of the nonlocal Davey-Stewartson I equation when $|\rho|>\rho_{0}$ and $\left|\lambda_{j}-\mathrm{i} \kappa_{j}\right|<\delta$ $(j=1, \cdots, n)$.

(i) $\widetilde{u}$ is globally defined and bounded for $(x, y, t) \in \mathbf{R}^{3}$.

(ii) Suppose the real numbers $v_{x}, v_{y}$ satisfy $\alpha_{k R} v_{x} \pm \beta_{k R} v_{y} \neq 0$ for all $k=1, \cdots, n$ where $\alpha_{k}$ 's and $\beta_{k}$ 's are given by (59), then $\lim _{s \rightarrow+\infty}|\widetilde{u}|=|\rho|$ along the straight line $x=x_{0}+v_{x} s$, $y=y_{0}+v_{y} s$ for arbitrary $x_{0}, y_{0} \in \mathbf{R}$.

Proof. (i) follows directly from Lemma 3. Now we prove (ii).

Since $\left|e_{k \pm}\right|=\mathrm{e}^{\left(\alpha_{k R} v_{x} \pm \beta_{k R} v_{y}\right) s+\left(\alpha_{k R} x_{0} \pm \beta_{k R} y_{0} \pm \gamma_{k R} t\right)}$ along the straight line $x=x_{0}+v_{x} s$, $y=y_{0}+v_{y} s, \alpha_{k R} v_{x} \pm \beta_{k R} v_{y} \neq 0$ implies that for each $k, e_{k+} \rightarrow 0$ or $e_{k+} \rightarrow \infty$, and $e_{k-} \rightarrow 0$ or $e_{k-} \rightarrow \infty$ as $s \rightarrow+\infty$. Let

$$
\begin{aligned}
& \mu_{k}= \begin{cases}\lambda_{k} & \text { if } \alpha_{k R} v_{x}+\beta_{k R} v_{y}>0, \\
-\lambda_{k}^{*} & \text { if } \alpha_{k R} v_{x}+\beta_{k R} v_{y}<0, \\
-\lambda_{k} & \text { if } \alpha_{k R} v_{x}-\beta_{k R} v_{y}>0, \\
\lambda_{k}^{*} & \text { if } \alpha_{k R} v_{x}-\beta_{k R} v_{y}<0,\end{cases} \\
& a_{k}= \begin{cases}\beta_{k} & \text { if } \alpha_{k R} v_{x}+\beta_{k R} v_{y}>0, \\
-\beta_{k}^{*} & \text { if } \alpha_{k R} v_{x}+\beta_{k R} v_{y}<0,\end{cases} \\
& b_{k}= \begin{cases}-\beta_{k} & \text { if } \alpha_{k R} v_{x}-\beta_{k R} v_{y}>0, \\
\beta_{k}^{*} & \text { if } \alpha_{k R} v_{x}-\beta_{k R} v_{y}<0,\end{cases}
\end{aligned}
$$


then

$$
a_{k}=\frac{1}{2}\left(\frac{\sigma|\rho|^{2}}{\mu_{k}}+\mu_{k}\right), \quad b_{k}=\frac{1}{2}\left(\frac{\sigma|\rho|^{2}}{\nu_{k}}+\nu_{k}\right) .
$$

Rewrite (15) as

$$
\left(\begin{array}{cccccc}
\left(G_{1}\right)_{11} & \cdots & \left(G_{n}\right)_{11} & \rho^{-1} \mathrm{e}^{\mathrm{i} \phi}\left(G_{1}\right)_{12} & \cdots & \rho^{-1} \mathrm{e}^{\mathrm{i} \phi}\left(G_{n}\right)_{12} \\
\left(G_{1}\right)_{21} & \cdots & \left(G_{n}\right)_{21} & \rho^{-1} \mathrm{e}^{\mathrm{i} \phi}\left(G_{1}\right)_{22} & \cdots & \rho^{-1} \mathrm{e}^{\mathrm{i} \phi}\left(G_{n}\right)_{22}
\end{array}\right) S W S^{-1}=-R S^{-1}
$$

where $S=\left(\begin{array}{cc}I_{n} & \\ & \rho \mathrm{e}^{-\mathrm{i} \phi} I_{n}\end{array}\right), I_{n}$ is the $n \times n$ identity matrix.

Applying Cramer's rule to (76) and using (75), we have

$$
\lim _{s \rightarrow+\infty} \widetilde{u}=\lim _{s \rightarrow+\infty}\left(\rho \mathrm{e}^{-\mathrm{i} \phi}+2\left(G_{1}\right)_{12}\right)=\rho \mathrm{e}^{-\mathrm{i} \phi}\left(1-2 \frac{\operatorname{det} W_{1}}{\operatorname{det} W_{0}}\right)=\rho \mathrm{e}^{-\mathrm{i} \phi} \frac{\operatorname{det} W_{2}}{\operatorname{det} W_{0}}
$$

where

$$
W_{0}=\left(\begin{array}{cccccc}
a_{1}^{n-1} & \cdots & a_{n}^{n-1} & \sigma|\rho|^{-2} b_{1}^{n-1} \nu_{1} & \cdots & \sigma|\rho|^{-2} b_{n}^{n-1} \nu_{n} \\
a_{1}^{n-2} & \cdots & a_{n}^{n-2} & \sigma|\rho|^{-2} b_{1}^{n-2} \nu_{1} & \cdots & \sigma|\rho|^{-2} b_{n}^{n-2} \nu_{n} \\
\vdots & & \vdots & \vdots & & \vdots \\
a_{1} & \cdots & a_{n} & \sigma|\rho|^{-2} b_{1} \nu_{1} & \cdots & \sigma|\rho|^{-2} b_{n} \nu_{n} \\
1 & \cdots & 1 & \sigma|\rho|^{-2} \nu_{1} & \cdots & \sigma|\rho|^{-2} \nu_{n} \\
a_{1}^{n-1} \mu_{1} & \cdots & a_{n}^{n-1} \mu_{n} & b_{1}^{n-1} & \cdots & b_{n}^{n-1} \\
a_{1}^{n-2} \mu_{1} & \cdots & a_{n}^{n-2} \mu_{n} & b_{1}^{n-2} & \cdots & b_{n}^{n-2} \\
\vdots & & \vdots & \vdots & & \vdots \\
a_{1} \mu_{1} & \cdots & a_{n} \mu_{n} & b_{1} & \cdots & b_{n} \\
\mu_{1} & \cdots & \mu_{n} & 1 & \cdots & 1
\end{array}\right)
$$

$W_{1}$ is obtained from $W_{0}$ by replacing the $(n+1)$-th row with

$$
\left(\begin{array}{cccccc}
a_{1}^{n} & \cdots & a_{n}^{n} & \sigma|\rho|^{-2} b_{1}^{n} \nu_{1} & \cdots & \sigma|\rho|^{-2} b_{n}^{n} \nu_{n}
\end{array}\right),
$$


and

$$
W_{2}=\left(\begin{array}{cccccc}
a_{1}^{n-1} & \cdots & a_{n}^{n-1} & \sigma|\rho|^{-2} b_{1}^{n-1} \nu_{1} & \cdots & \sigma|\rho|^{-2} b_{n}^{n-1} \nu_{n} \\
a_{1}^{n-2} & \cdots & a_{n}^{n-2} & \sigma|\rho|^{-2} b_{1}^{n-2} \nu_{1} & \cdots & \sigma|\rho|^{-2} b_{n}^{n-2} \nu_{n} \\
\vdots & & \vdots & \vdots & & \vdots \\
a_{1} & \cdots & a_{n} & \sigma|\rho|^{-2} b_{1} \nu_{1} & \cdots & \sigma|\rho|^{-2} b_{n} \nu_{n} \\
1 & \cdots & 1 & \sigma|\rho|^{-2} \nu_{1} & \cdots & \sigma|\rho|^{-2} \nu_{n} \\
-\sigma|\rho|^{2} a_{1}^{n-1} \mu_{1}^{-1} & \cdots & -\sigma|\rho|^{2} a_{n}^{n-1} \mu_{n}^{-1} & -\sigma|\rho|^{-2} b_{1}^{n-1} \nu_{1}^{2} & \cdots & -\sigma|\rho|^{-2} b_{n}^{n-1} \nu_{n}^{2} \\
a_{1}^{n-2} \mu_{1} & \cdots & a_{n}^{n-2} \mu_{n} & b_{1}^{n-2} & \cdots & b_{n}^{n-2} \\
\vdots & & \vdots & \vdots & & \vdots \\
a_{1} \mu_{1} & \cdots & a_{n} \mu_{n} & b_{1} & \cdots & b_{n} \\
\mu_{1} & \cdots & \mu_{n} & 1 & \cdots & 1
\end{array}\right) .
$$

Denote $\mathrm{ROW}_{k}$ and $\mathrm{COL}_{k}$ to be the $k$-th row and $k$-th column of $W_{2}$ respectively. The elementary transformations

$$
\begin{aligned}
& \mathrm{ROW}_{n+k+1}-2 \cdot \mathrm{ROW}_{k} \rightarrow \mathrm{ROW}_{n+k+1} \quad(k=1, \cdots, n-1), \\
& \mu_{k} \cdot \mathrm{COL}_{k} \rightarrow \mathrm{COL}_{k} \quad(k=1, \cdots, n), \\
& \sigma|\rho|^{2} \nu_{k}^{-1} \cdot \mathrm{COL}_{n+k} \rightarrow \mathrm{COL}_{n+k} \quad(k=1, \cdots, n), \\
& -\sigma|\rho|^{-2} \cdot \mathrm{ROW}_{n+k} \rightarrow \mathrm{ROW}_{n+k} \quad(k=1, \cdots, n), \\
& \mathrm{ROW}_{k} \leftrightarrow \mathrm{ROW}_{n+k} \quad(k=1, \cdots, n)
\end{aligned}
$$

transform $W_{2}$ to $W_{0}$. Hence $\operatorname{det} W_{2}=\prod_{k=1}^{n} \frac{\nu_{k}}{\mu_{k}} \operatorname{det} W_{0}$. This leads to $\lim _{s \rightarrow+\infty}|\widetilde{u}|=|\rho|$ since $\prod_{k=1}^{n}\left|\mu_{k}\right|=\prod_{k=1}^{n}\left|\nu_{k}\right|=\prod_{k=1}^{n}\left|\lambda_{k}\right|$. The theorem is proved.

A 2 "line dark soliton" solution is shown in Figure 4 where the parameters are $\sigma=-1$, $t=10, \rho=1, \lambda_{1}=0.8+0.1 \mathrm{i}, \lambda_{2}=-0.6-0.3 \mathrm{i}$. The figure on the right describes the same solution but is upside down.

\section{Acknowledgements}

This work was supported by the Natural Science Foundation of Shanghai (No. 16ZR1402600) and the Key Laboratory of Mathematics for Nonlinear Sciences of Ministry of Education of China. The author is grateful to Prof. S.Y.Lou for helpful discussion. 


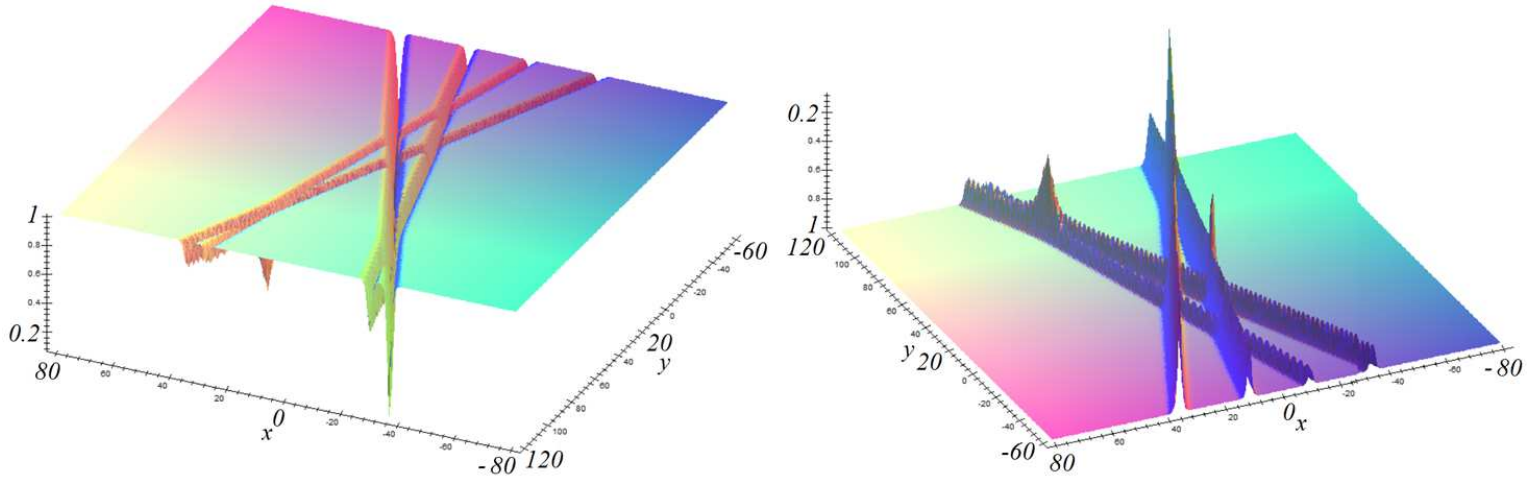

Figure 4: $|\widetilde{u}|$ of a 2 "line dark soliton" solution.

\section{References}

[1] Ablowitz M J and Musslimani Z H 2013 Integrable nonlocal nonlinear Schrödinger equation, Phys. Rev. Lett. 110, 064105

[2] Ablowitz M J and Musslimani Z H 2016 Inverse scattering transform for the integrable nonlocal nonlinear Schrödinger equation, Nonlinearity 29, 915-946

[3] Ablowitz M J and Musslimani Z H 2016 Integrable nonlocal nonlinear equations, https://arxiv.org/abs/1610.02594

[4] Gadzhimuradov T A and Agalarov A M 2016 Towards a gauge-equivalent magnetic structure of the nonlocal nonlinear Schrödinger equation, Phys. Rev. A 93, 062124

[5] Huang X and Ling L M 2016 Soliton solutions for the nonlocal nonlinear Schrödinger equation, Eur. Phys. J. Plus 131, 148

[6] Khare A and Saxena A 2015 Periodic and hyperbolic soliton solutions of a number of nonlocal nonlinear equations, J. Math. Phys. 56, 032104

[7] Konotop V V, Yang J and Zezyulin D A 2016 Nonlinear waves in PT-symmetric systems,, Rev. Mod. Phys. 88, 035002

[8] Li M and Xu T 2015 Dark and antidark soliton interactions in the nonlocal nonlinear Schrödinger equation with the self-induced parity-time-symmetric potential, Phys. Rev. E 91, 033202

[9] Lou S Y 2016 Alice-Bob systems, $P_{s^{-}} T_{d^{-}} C$ principles and multi-soliton solutions, https://arxiv.org/abs/1603.03975.

[10] Ma L Y and Zhu Z N $2016 N$-soliton solution for an integrable nonlocal discrete focusing nonlinear Schrödinger equation, Appl. Math. Lett. 59, 115-121 
[11] Sarma A K, Miri M A, Musslimani Z H and Christodoulides D N 2014 Continuous and discrete Schrödinger systems with parity-time-symmetric nonlinearities, Phys. Rev. E 89, 052918

[12] Fokas A S 2016 Integrable multidimensional versions of the nonlocal nonlinear Schrödinger equation, Nonlinearity 29, 319-324

[13] Salle M A, Leble S B and Yurov A V 1989 in Nonlinear World, Proc. of the IV Int. Workshop on Nonlinear and Turbulent Processes in Physics 2, Ed. Sitenko A G, Zakharov V E (Naukova Dumka, Kiev) 287-291

[14] Matveev V B and Salle M A 1991 Darboux transformations and solitons (Berlin: Springer)

[15] Boiti M, Leon J P and Pempinelli F 1990 Multidimensional solitons and their spectral transforms, J. Math. Phys. 31, 2612-2618

[16] Heredero R H, Alonso L M and Reus E M 1991 Fusion and fission of dromions in the Davey-Stewartson equation, Phys. Lett. A 152, 37-41

[17] Hietarinta J and Hirota R 1990 Multidromion solutions to the Davey-Stewartson equation, Phys. Lett. A 145, 237-244

[18] Zhou Z X 1988 On the Darboux transformation for 1+2 dimensional equations, Lett. Math. Phys. 16, 9-17

[19] Gu C H, Hu H S and Zhou Z X 2005 Darboux Transformations in Integrable Systems (Dordrecht: Springer) 